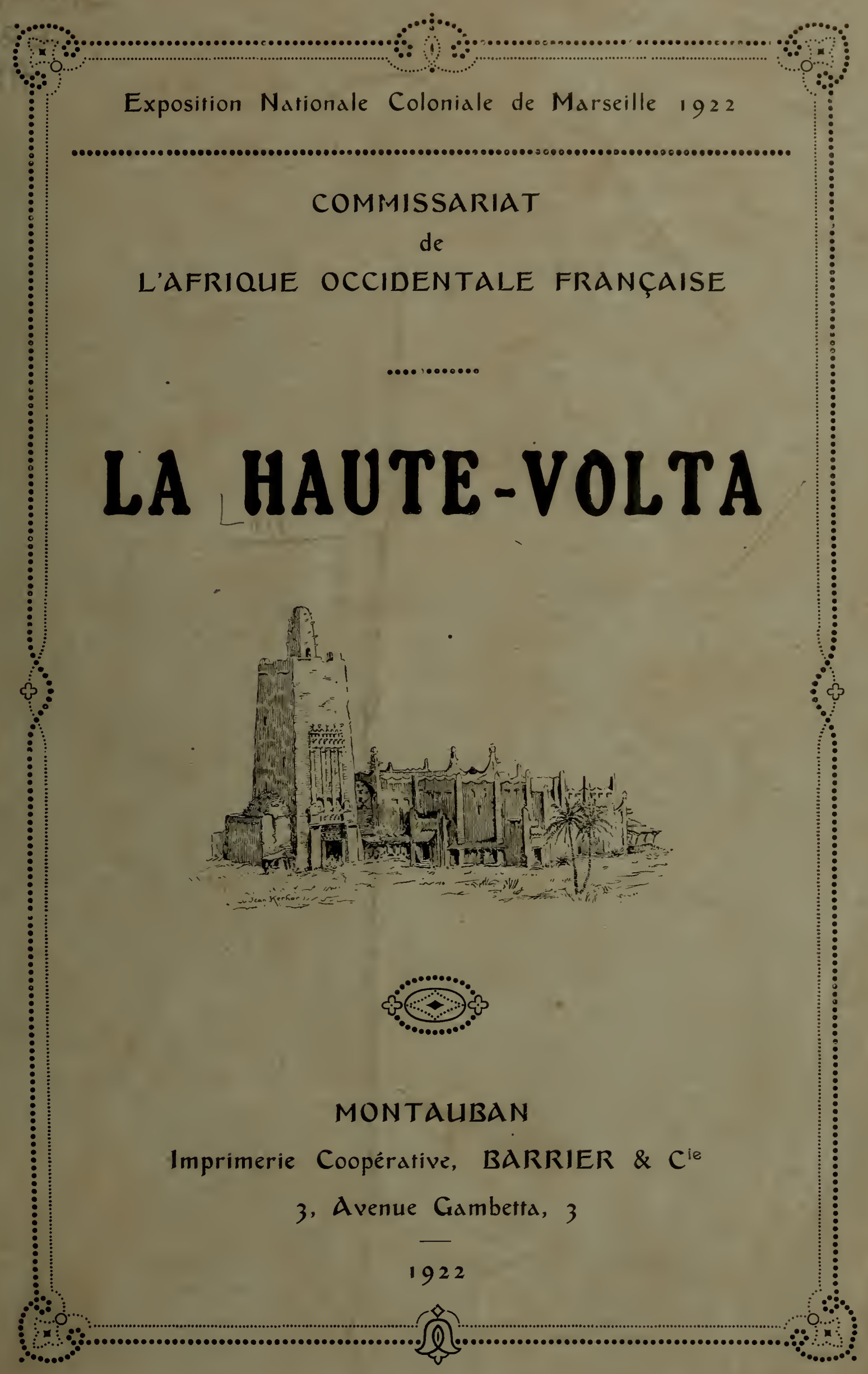





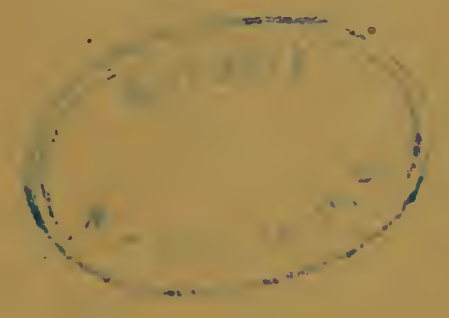


Les personnes qui, après avoir pris connaissance de ce tract, désireraient une documentation plus complète, sont priées de s'adresser à l'Agence Économique de l'Afrique Occidentale Française, 2\%, boulevard des Italiens, à Paris, qui leur fournira immédiatement, soit oralement, soit par écrit, tous les renseignements nécessaires et toutes les indications utiles. 


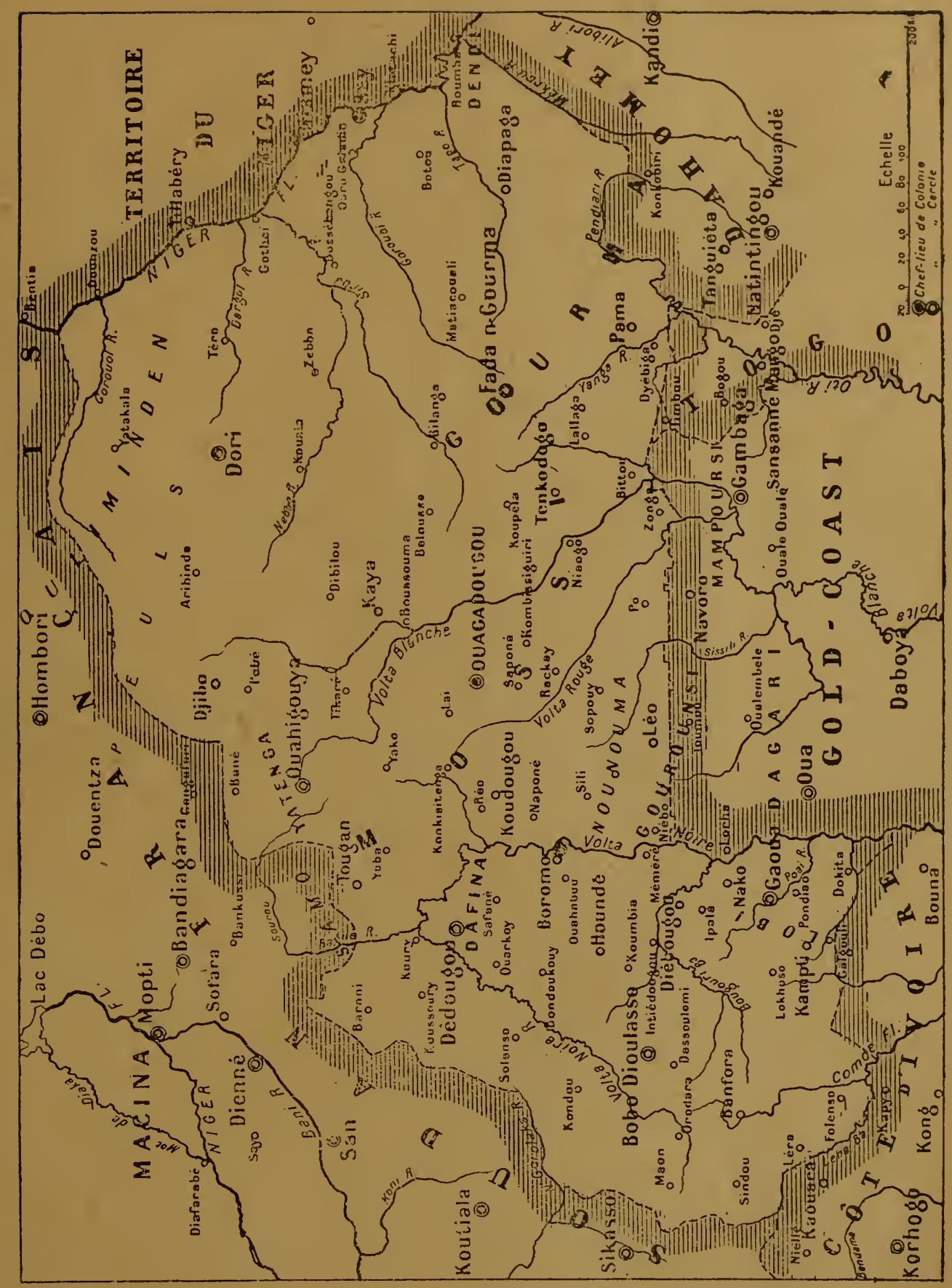





\section{LA HAUTE-VOLTA}

\section{CHAPITRE PREMIER}

\section{Le Pays, le Climat, les Habitants}

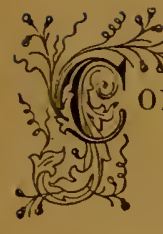

\section{Le Pays}

Bandiagara), à l'est par le Moyen-Niger qui la sépare du Territoire du Niger (Cercle de Niamey), au sud par le Dahomey, le Togo, la Cold Coast et la Côte d'Ivoire, à l'ouest par le Soudan français (Cercles de Sikassio, Koutiala et San).

Deux régions naturelles correspondant aux bassins du Hautet Moyen-Niger et de la Volta constituent la Haute-Volta. Le premier, le moins important, comprend les Cercles de Ouahigouya et Dori; il se réduit à quelques cours d'eau insignifiants, lits desséchés pendant la majeure partie de l'année, torrentiels aux heures qui suivent immédiatement les fortes chutes des pluies d'hivernage, en tout temps impraticables à la navigation. L'aspect du pays est celui des zones sahéliennes, caractérisé par de vastes espaces sablonneux où pousse une végétation rabougrie principalement composée d'épineux et d'arbres à gomme.

Le deuxième bassin qui s'étend sur la plus grande surface de la colonie est représenté par les trois Volta, la Noire, la Rouge, 
et la Blanche et par l'Oti, tous quatre affluents du fleuve Volta qui se jette dans l'Atlantique entre Lomé et Accra. Tous ces cours dieau sont alimentés par les pluies de l'hivernage et se réduisent pendant la saison sèche à une série de mares isolées. L'aspect du pays, sans être encore très luxuriant est égayé, surtout pendant la période des pluies, par d'immenses champs de mil que l'humidité du sol et sa teneur en humus permettent aux indigènes de cultiver en grand. La végétation arborescente $\mathrm{y}$ est faible, sauf dans les thalwegs où l'on rencontre de beaux arbres.

De l'ensemble des fleuves et des rivières qui arrosent la HauteVolta, le Niger et la Volta Noire méritent seuls une mention spéciale. La Volta Noire qui sépare les Cercles du Lobi et de Dédougou a été jadis utilisée comme moyen de transport sur une notable partie de son parcours et sa navigabilité n'est qu'une question d'étude qui pourra être envisagée ultérieurement.

Le Niger suit la frontière orientale de la colonie sur une tongueur de 400 kil. environ. A sa sortie du Soudan français il se trouve encaissé entre ses deux rives et, pendant une centaine de kilomètres, en amont de Dounzou, est coupé par des rapides, principalement les rapides de Fafa et de Lubézenga. Accessible aux chalands de 4 à 6 tonnes à l'époque des hautes eaux (octobreavril), la navigation n'est possible durant la période sèche et dans certaines parties que pour les pirogues de faible tirant d'eau.

\section{Le Climat}

Si l'on met à part les Cercles de Dori et de Ouahigouya, appartenant à la région sahélienne et, comme tels, doués d'uno température presqu'uniformément chaude, sèche le jour et fraî. che la nuit, on peut distinguer dans la partie moyenne et méridionale de la colonie deux saisons principales : la saison sèche et la saison des pluies.

La saison sèche va du début de novembre à fin mai. Jusqu'au début de février la température est relativement fraîche; elle devient torride de février à mai. La saison des pluies va de juin à fin octobre; elle est chaude et humide. Les pluies y sont particulièrement abondantes de la fin du mois de juin à mi-septembre. Elles sont accompagnées le plus souvent de violents orages où le tonnerre et le vent se déchaînent avec furie. 
La hauteur d'eau tombée annuellement va de $0 \mathrm{~m}$. 40 à 3 mètres environ suivant les régions. Toujour's suffisante dans les régions du sud, sa répartition est, pour les Cercles du nord, d'une importance capitale, car c'est d'elle que dépend le sort des cultures toutes hàtives dont quatre mois à peine séparent les emblavements de la maturation.

Le nombre des jour's de pluie varie de 25 à 75 . L'hygrométrie oscille, avec les saisons, entre 40 et 90.

Les différences de température sont plus sensibles dans le nord que dans le sud. Dans la zone sahélienne le froid est plus vif en hiver et la chaleur plus forte en saison sèche. La moyenne des minima est de $16^{\circ}$ et celle des maxima de $30^{\circ}$.

Les vents dominants sont ceux du nord-est (Alizés ou Harmattan), vents très chauds et desséchants. La Mousson souffle au début de la saison fraîche et à la fin de la saison chaude, mais se fait assez peu sentir. Le vent dles tornades est toujours orienté est-ouest.

Dans son ensemble le climat de la Haute-Volta est favorable à l'européen qui peut y faire des séjours prolongés, sous la réserve qu'il s'astreigne aux exigences de l'hygiène tropicale.

\section{Les Habitants}

La Haute-Volta compte environ 3 millions d'habitants disséminés sur une superficie de 300.000 kilomètres carrés, soit dix habitants au kilomètre carré. Très uense dans la région du Mossi, la population devient plus clairsemée dans l'ouest; la sècheresse résultant du climat ou l'absence de points dieau la rend parfois très faible ou nulle dans les Cercles du nord et de l'est qui comptent de grands espaces à peu près désertiques.

Les races qui peuplent la Haute-Volta peuvent se diviser en deux blocs principaux : races voltaïques ou aborigènes qui comprennent le groupe Mossi, le groupe Gourounsi, le groupe Bobo et quelques autres groupes moins importants; et races envahissantes qui englobent le groupe Peuhl, le groupe Soninké, le groupe Songhraï, et quelques autres groupes moins considérables.

Les races voltaïques et les races envahissantes se différencient d'une manière générale par plusieurs caractères dont les plus remarquables sont les suivants : 
$1^{\circ}$ Les premières sont fétichistes tandis que les autres pratiquent la religion musulmane;

$2^{\circ}$ Les Voltaïques s'adonnent en grosse majorité à l'agriculture tandis que les musulmans, commerçants ou pasteurs, vivent des échanges ou de l'élevage;

$3^{\circ}$ Les Voltaïques sont établis dans les Cercles de Ouagadougou, Gaoua, Bobo-Dioulasso, Dédougou et Ouahigouya; l'habitat des musulmans est limité essentiellement aux Cercles de Say et de Dori.

\section{Races Aborigènes}

Groupe Mossi. - Ce groupe compte environ 1.700000 représentants. Bien que parlant des dialectes assez différents, les indigènes qui le composent ont gardé entre eux des affinités au point de vue physique, religieux et moral.

Le Mossi présente déjà un type plus affiné que le noir ordinaire : de taille moyenne, les traits plus délicats, de structure solide, il a le grave défaut de s'adonner à l'alcool. Une notable partie de la récolte de grains est consacrée à la confection d'une bière spéciale qui, dans certaines régions, constitue la base de l'alimentation indigène. Cette consommation excessive de boissons fermentées jointe aux disettes qui, dans le nord, ont pu résulter d'années d'une exceptionnelle sècheresse explique l'état stationnaire d'une population par ailleurs intéressante et laborieuse.

Groupe Gourounsi. - Ce groupe compte environ 400.000 individius. Le Gourounsi présente de nombreuses analogies avec le Mossi, mais, demeuré plus primitif, il vit dans une sorte d'état anarchique, ignorant de toute autorité. La franchise de son caractère et son état de prévoyance distingue le Gourounsi' et le rendent, à ce double point de vue, bien supérieur à son voisin Mossi. En pays Gourounsi, la règle, qui est strictement observée, est de ne jamais toucher aux produits de la récolte avant une année écoulée : cette mesure explique les réserves considérables, datant parfois de plusieurs hivernages, en dépôt dans les villages.

Groupe Bobo. - Ge groupe dont l'habitat se confond avec les limites mêmes du Cercle de Bobo-Dioulasso et quelques cantons du sud-ouest de Dédoug:ou compte environ 376.000 individus. Ses traits distinctifs ne sont guère différents de ceux du Mossi 


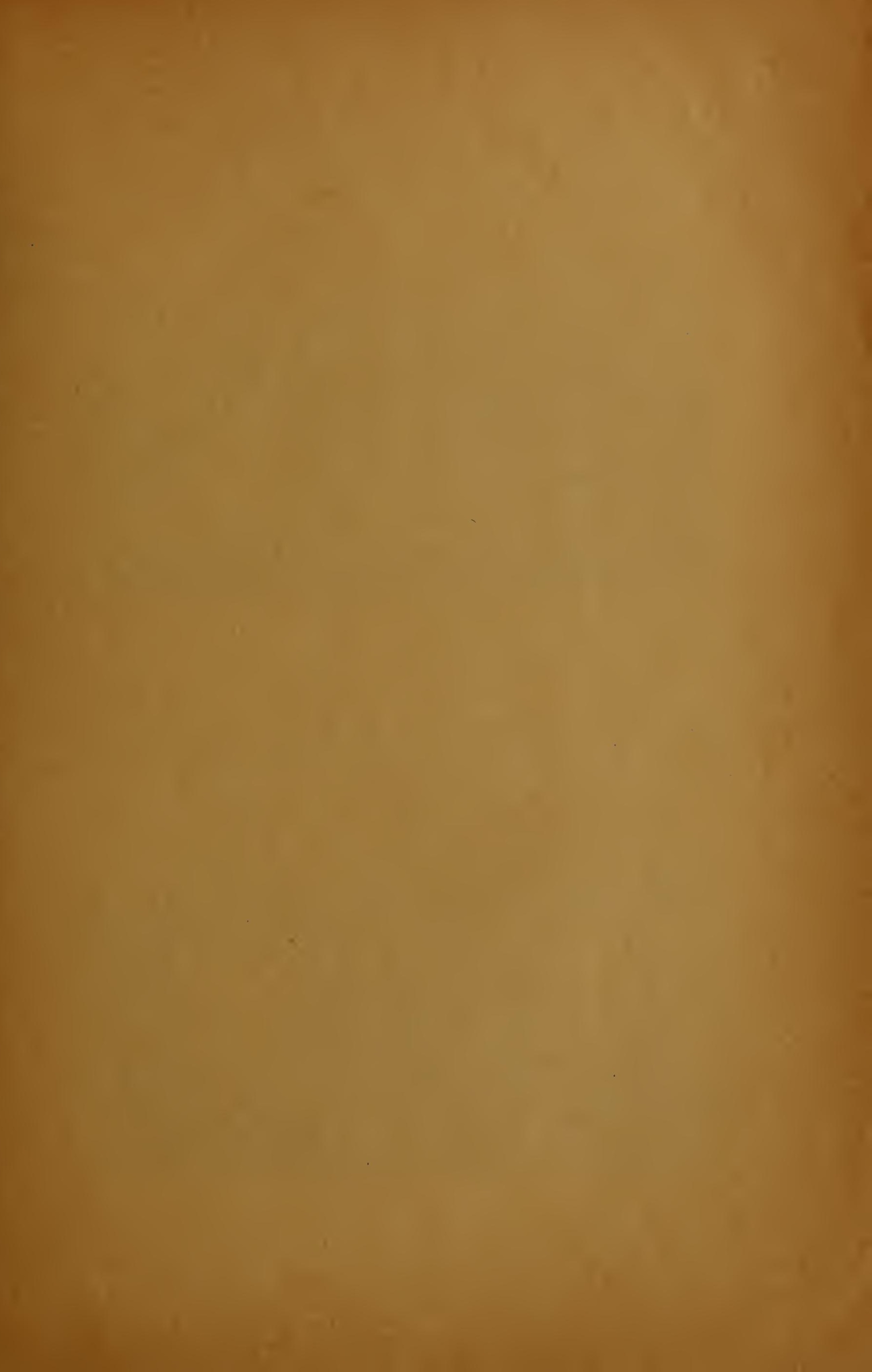




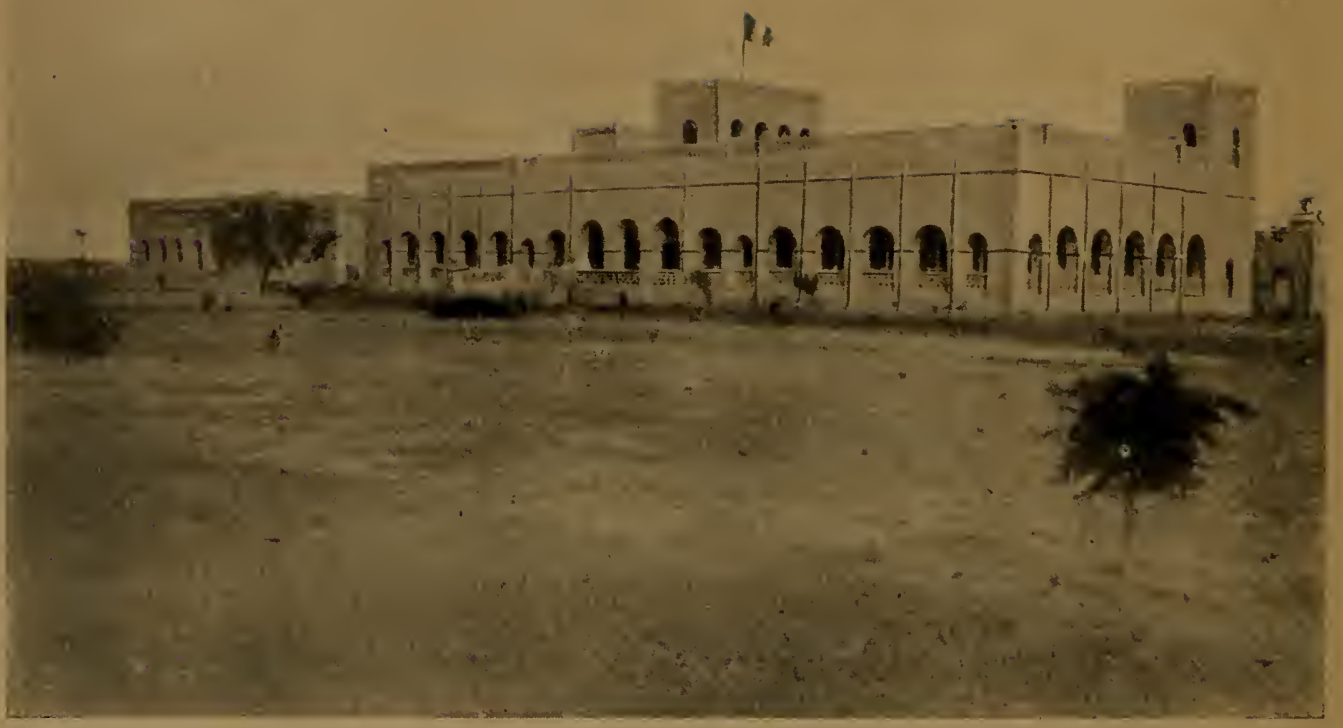

Fig. 1. - Ouagadougrou : Le Gouvernement.

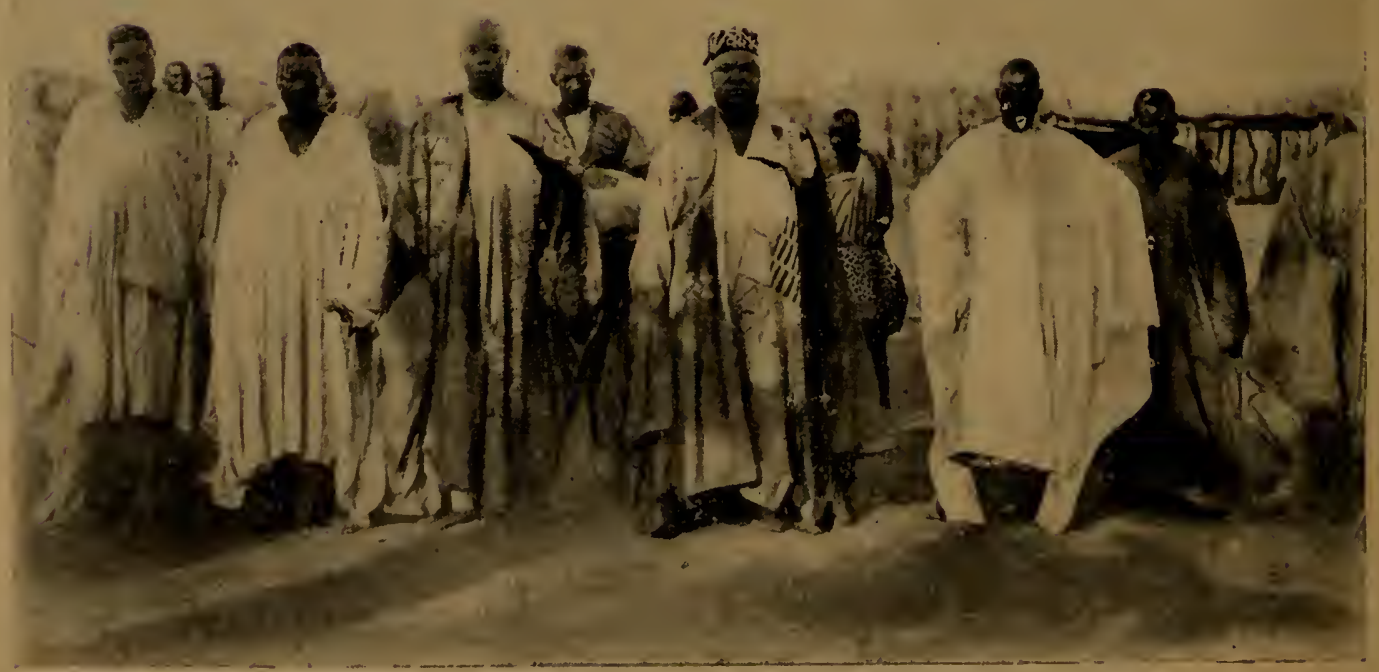

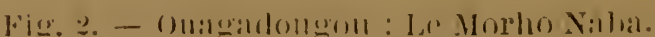


et Gourounsi. Le caractéristique du Bobo réside dans son amour de la terre et ses aptitudes remarquables pour la culture : les campagnes bobos, naturellement fertiles, offrent, dans la saison des travaux agricoles, l'aspect de vastes jardins entretenus avec le soin le plus méticuleux.

Groupes divers. - Ce sont les Samos de Dédougou, raco quelque peu turbulente et avide d'indépendance; les Sia ainsi désignés du nom de la ville de Bobo-Dioulasso; les Baribas de Fada; les Bellahas de Dori, etc., représentant à eux tous un total d'environ 70.000 habitants.

\section{Races Envahissantes}

Groupe Peuhl. - Les familles peuhles qui peuplent la HauteVolta proviennent toutes du Fouta-Toro sur la rive gauche du Sénégal ou des environs de Kayes; leur arrivée dans la colonie date des $\mathrm{XVI}^{\circ}$ et $\mathrm{XVII}{ }^{\circ}$ siècles, après plusieurs arrêts dans le Macina et l'Issa-Ber. Cet élément est représenté par environ 310.000 individus répartis dans tous les Cercles; groupés dans la région du nord où ils se livrent à l'élevage pour leur propre compte, ils se rencontrent aussi dans les Cercles du sud où ils sont bergers à la solde des riches Mossi.

Lo Peuhl de la Haute-Volta est sédentarisé; en saison sèche, il descend avec ses troupeaux vers les pâturages du sud demeu. rés plus abondants. Il s'adonne aussi à l'agriculture, mais dans la mesure rigoureuse de sa consommation.

La question de l'origine des Peuhls n'est pas éclaircie : leur type physique qui se rapproche de l'Européen, leurs traits réguliers et d'une extrême finesse, leur crâne allongé, leur visage ovale, leur teint qui varie du noir au rouge clair, leur langue et leurs mœurs ont permis de les rattacher au groupe sémite de la race caucasique.

Groupe Soninké. - Ce groupe se rattache à la famille des Soninkés du Soudan français. Les 115.000 individus qui le composent sont tous concentrés dans le Cercle de Dédougou. Le Soninké a les yeux tirés et ses pommettes saillantes révèlent son origine asiatique. Sa langue est un idiome mandé très altéré.

Groupe Songhraï. - Le type Songhraï présente quelques affinités physiques avec les Peuhls; il a une ligne moins élégante 
mais il est plus robuste. Certaines pratiques telles que l'embaumement font supposer que les Songhraï viennent de la région du Nil ou qu'ils ont été en contact avec les Egyptiens.

Les Cercles de Dori (42.000) et de Say (8.000) se partagent les 50.000 individus qui comprennent ce groupe.

Groupe Dioula. - Les 40.000 individus qui composent ce groupe sont venus de la haute Côte-d'Ivoire avec les conquérants Ouattaras installés à Bobo-Dioulasso.

Groupes divers. - (Touaregs, Haoussas). L'ensemble de ces groupes forme un total approximatif de 20.000 individus dont 5.000 Touaregs.

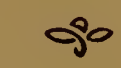

D'une façon générale, la famille est partout fortement constituée avec une organisation analogue à la " gens " romaine. C'est le régime patriarcal : tous les membres d'une mème famille sont sous l'autorité du chef qui est le représentant le plus âgé de la branche aînée. Ce "Pater familias " est à la fois le chef, le juge et le prêtre de la maison : lui seul détient tous les pouvoirs pour tous les actes de la vie; notamment, c'est lui et non le père qui donne les autorisations de mariage. Il tranche également tous les différends qui peuvent surgir au sein de la famille. Dans toutes ces décisions, il n'est lié que par la coutume qu'il est tenu de respecter. Enfin, à lui revient l'honneur d'offrir les sacrifices dans les occasions solennelles.

La polygamie est la règle de toutes ces populations, sauf chez les Touaregs et les Mossi catholiques. Le nombre des femmes n'a d'autres limites que les ressources du mari qui a une tendance marquée à ne considérer ses épouses que comme des domestiques et des procréatrices l'enfants dont le travail, les dots les dons coutumiers constitueront à leur tourpour lui une source de revenus. On voitque la condition de la femme est très inférieure ; elle s'améliore cependant chaque jour sous notre action par suite de la suppression des châtiments corporels, et en raison des facilités qui sont données aux femmes d'entamer la procédure du divorce, quand elles sont maltraités par leurs maris. 



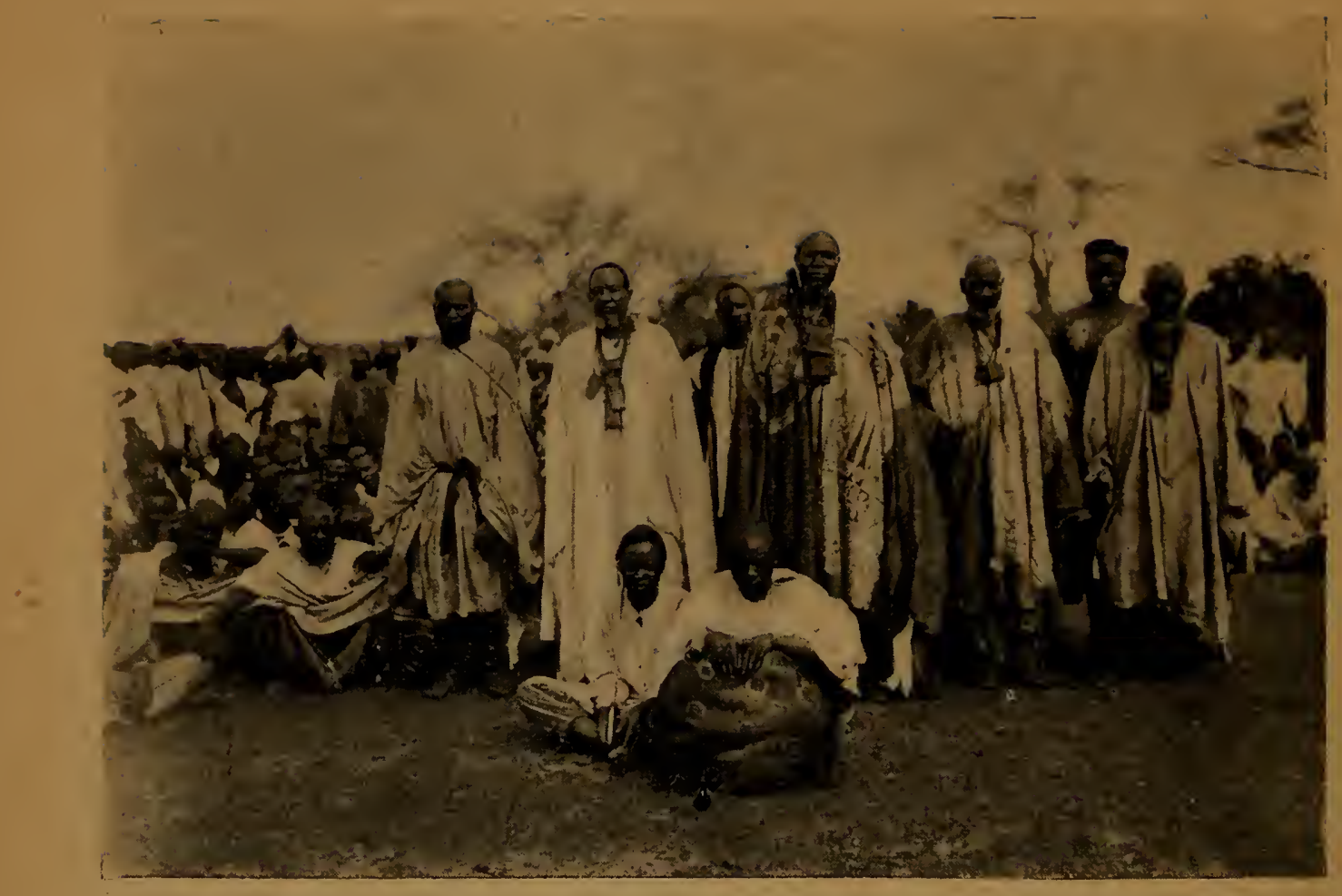

Fig. 3. - Ouahigouya : Le Yatanya-Naba

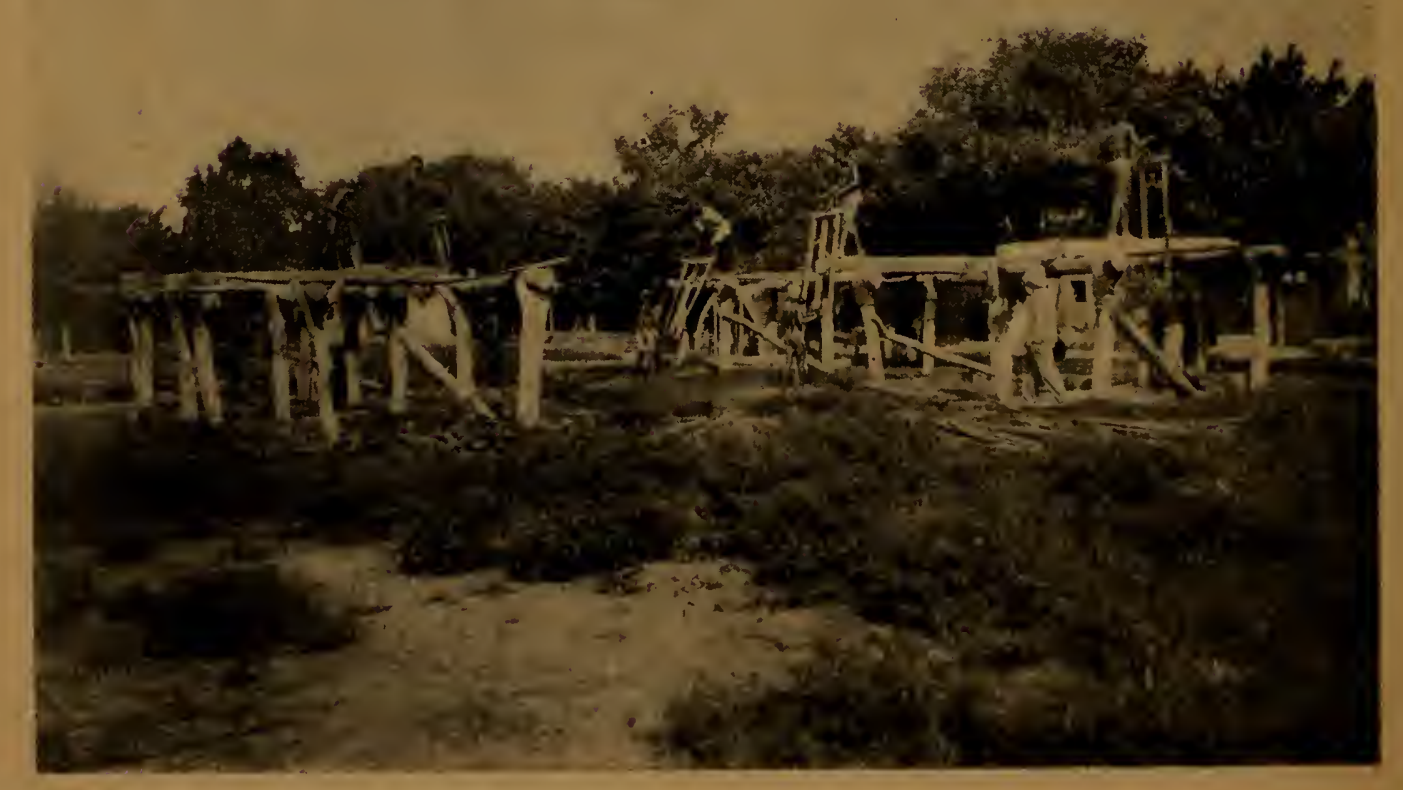

Fig. 1. - onagadongou : scirurs he long.

IIUTE-VOLTA. - PLANCHE II 


\section{CHAPITRE II}

\section{La Conquête du Pays}

Les relations du Mossi avec l'Europe avant notre arrivée sont plutôt du domaine de la légende et ne reposent sur aucune donnée précise. Des auteurs parlent toujours des relations de l'empereur du Mossi avec Jean II du Portugal et même Jean III, vers le XVI ${ }^{\mathbf{e}}$ siècle; maís, ni l'histoire, ni la tradition locale, ne confirment cette version. Nous devons attendre jusqu'au milieu du $\mathrm{XIX}^{\bullet}$ siècle pour obtenir les premiers renseignements sur la partie nord du pays visité en 1853 par Barth qui parcourut le Yaga et le Djennaré. L'exploration en 1887-1889, par Binger, de BoboDioulasso et du Mossi a laissé un souvenir très vivace dans l'esprit des indigènes. En 1890-91 la mission Monteil traversa la boucle du Niger pour aboutir à Say d'où son voyage se poursuivit vers la Méditerranée par le Tchad et Tripoli.

Ce fut en 1895 que d'une façon effective la France prit contact avec la Haute-Volta; le mûlatre anglais Ferousson, au service de l'Angleterre, se dirigeait vers le Mossi quand le Colonel de Trentinian, gouverneur du Soudan, et M. Ballot, gouverneur du Dahomey, sentant le danger d'une prise de possession par cet agent britannique de cet hinterland momentanément neutralisé, ce qui eût entraîné l'isolement de nos deux colonies, prirent les mesures nécessaires pour le ldevancer. Le Commandant Destenave, résident de Bandiagara, 'notre poste le plus avancé dans l'est à cette époque, reçut l'ordre d'occuper la région contestée. Il entra à Ouahigouya et à Dori, se rendit à Yako malgré la résistance du Naba, mais se vit refuser l'accès de Ouag̣adougou. En mai 1885, le Colonel de Trentinian donne mission au Lieutenat Voulet de devancer les anglais qui, aidés de Samory, menaçaient le Mossi, puis, cette tâche terminée, de relier le Mossi au Dahomey. Le 30 juillet 1896, le Lieutenant Voulet, assisté du Lieutenant Chanoine, quittait Bandiagara à la tête d'une colonne de 50 tirailleurs, passait à Ouahigouya, recevait la soumission du Naba de Yako, puis le $1^{\text {er }}$ septembrecampait à 
Ouagadougou.Kouka-Kountou nommé Empereur du Mossi sous le nom de Naba Tigri en remplacement du Naba Wobre des. titué, passait le 20 janvier 1897 avec le Lieutenant Voulet un traité qui plaçait le Mossi sous le protectorat français.

La majeure partie de la Haute-Volta était réduite, il ne restait plus à occuper que le pays Gourounsi, le pays Bobo et une portion du Cercle actuel de Dédougou. L'année 1897 vit. la soumission de Gourounsi par le Capitaine Scal; la même année les régions de Dédougou et Diébougou reconnaissaient notre autorité. Enfin le grand territoire de Bobo-Dioulasso qui vivait sous le sceptre des Ouattaras tombait au pouvoir dhu Commandant Caudrelier, établi en 1898 dans la ville-même de Bobo.

L'année 1902 qui marqua la création d'un poste à Gaoua, au centre du Lobi, peut être considérée comme la dernière étape de nos opérations militaires de conquête. Tous les territoires ainsi placés sous notre \&utorité firent partie intégrante du Haut-Sénégal et Niger jusqu'au $1^{\text {er }}$ mars 1919 où ils furent érigés en une colonie autonome sous le nom de Haute-Volta.

\section{CHAPITRE III}

\section{Situation politique et administrative actuelle}

La colonie de la Haute-Volta, créée par décret du $1^{\text {er }}$ mars 1919 a été constituée par la réunion d'un certain nombre de Cercles détachés de l'ancien Haut-Sénégal.- Niger, aujourd'hui Soudan français. Le chef-lieu sst Ouagadougou où réside un Lieutenantgouverneur représentant le Gouverneur général de l'Afrique Occidentale Française. Ouagadougou est le siège du $6^{\mathrm{C}}$ bataillon de tirailleurs sénégalais. Le calme le pluscomplet règrne dans cess régions et, là comme ailleurs, quelques détachements de gardes de Cercle suffisent aux administrateurs pour assurer la sécurité du pays et l'exécution de leurs ordres.

Le service de la Justice européenne est représenté par une Justice de paix à compétence étendue dont le ressort s'étend à tout le territoire de la colonie.

La justice indigène est rendue par des tribunaux de subdivisions statuanten premier ressort sur toutes les affaires civiles et 
correctionnelles ainsi que surcertaines infractions limitativement déterminées; les tribunaux de cercle connaissent des mèmes affaires en appel et des affaires crininelles en premier ressort; les différentes juridictions où le statut des justiciables est obligatoirement respecté, inspirent pleine confiance à nos administrés qui s'adressent à elles pour la solution de leurs litiges; les magistrats indigènes choisis parmi les notables les plus dignes s'acquittent de leurs fonctions ávec tact, conscience, impartiaiıté. Indépendamment du contrôle exercé sur la justice par le Chef de lil colonie et le Parquet général, la Chambre spéciale d'homologation de la Cour d'Appel del'A. O.F., sorte de cour de Cassation en l'espèce, examine tous les dossiers comportant des condamnations supérieures à cinq années de prison et homolog'ue ou renvoie les affaires appelées à être jugées à nouveau.

Guidée par le souci du bien-ètre matériel de la population placeée sous notre tutelle, l'Administration n'a pas négligé les mssures propres à assurer l'hygiène et la santé publiques. Dans cet ordre d'idées ont été créés un Service de santé et un Comité d'hygiène. Autant que les ressources du personnel l'ont permis, les Cercles les plus importants ont été pourvus de médecins qui, chaque jour, soit au chef-lieu du Cercle, soit au cours de leurs tournées, dionnent leurs soins gratuits aux indigènes qui arrivent toujours plus nombreux à la consultation .Il y a lieu de compter que dans un avenir prochain, tous les postes se verront dotés de médecins, l'auxiliaire le plus précieux de notre action civilisatrice. En attendant cette réalisation, Cercles et Subdivisions possèdent un dispensaire avec infirmiers vaccinateurs, pharmacie complète et le minimum d'outillage nécessaire.

Le service zootechnique est en voie d'organisation. Son fonc. tionnement est destiné à rendre d'inappréciable services dans un pays dont le cheptel constitue la principale. richesse, susceptible d'une extension indéfinie. Des vaccinateurs vétérinaires, formés au laboratoire zootechnique de Bamako surveillent les troupeaux et sous la direction du vétérinaire-chefdu service et des adminis trateurs, avisent aux mesures que comportent les circonstances.

Le service de l'agriculture provisoirement aux mains du Directeur de l'école régionale de Ouagadougou, dispose d'une école pour la formation des jardiniers, maraichers et pépiniéristes. A bref délai viendront des agents techniques qui auront mission 
d'étudier les cultures propres à la mise en valeur, suivant des conceptions rationnelles adaptées au pays, des espaces immenses qui ne réclament qu'un effort ingénieux pour décupler le rendement des produits les plus variés.

Le développement de l'enseignement a retenu l'attention du Gouvernement de la Haute-Volta. La colonie est déjà dotée d'un personnel européen et indigène à la hauteur de la tâche, entre toutes importante, qui lui incombe. Ouagadougou qui avait déjà une école primaire et une école régionale a vu ses institutions renforcées et complétées par la création d'une école primaire supérieure. Liéducation technique se trouve assurée par une école de charronnage destinée à faire des ouvriers charrons, par une école de pontonniers qui fournira aux cercles des ouvriers expérimentés pour la construction des routes et notamment des travaux d'art, par une école professionnelle où l'administration, le commerce et l'industrie trouveront des agents sérieux. Les écoles régionales ont été multipliées; le recrutement des écoles des villages a été intensifié et sélectionné. Des écoles de filles ont été prévues. Partout où réside un agent de l'autorité fonctionne une école. Les plus grands espoirs reposent sur cette politique scolaire qui est appelée à créer une élite sociale dans le pays et à favoriser l'évolution des indigènes dans la voie du progrès.

\section{Tableaux Récapitulatifs de la Population}

Population Indigène par Cercle et par Religion

\begin{tabular}{|c|c|c|c|c|}
\hline CERGLES & FÉtICHISTES & MUSULMaNS & CATHOLIQUES & TOTAUX \\
\hline Mossi & 1.160 .000 & 112.000 & 3.000 & 1.275 .000 \\
\hline Dédougou & 279.000 & 143.000 & » & 422.000 \\
\hline Bobo. & 268.000 & 42.000 & 》) & 310.000 \\
\hline Ouahigouya . & 211.300 & 38.700 & ” & 250.000 \\
\hline Fada n'Gourma & 179.700 & 10.300 & ") & 190.000 \\
\hline Saour. & 158.000 & 2.000 & ” & 160.000 \\
\hline Dori & 11.000 & 119.000 & ” & 130.000 \\
\hline Say & 9.000 & 60.000 & ” & 69.000 \\
\hline Tenkodogo.. & 267.000 & 8.000 & ” & 275.000 \\
\hline Total. & 2.543 .000 & 535.000 & 3.000 & 3.081 .000 \\
\hline
\end{tabular}


Population Européenne

\begin{tabular}{|c|c|c|c|c|}
\hline NATIONALITÉS & HOMMES & FEMMES & ENFANTS & TOTAUX \\
\hline Français & 98 & 23 & 6 & 127 \\
\hline Américains.. & 3 & 5 & " & 8 \\
\hline Syriens & 1 & $\because$ & ) & 1 \\
\hline Total. & 102 & 28 & 6 & 136 \\
\hline
\end{tabular}

\section{CHAPITRE IV}

\section{Voies de Communications et Moyens de Transports}

Les moyens decommunications et de transports de la HauteVolta tant extérieurs qu'intérieurs peuvent se diviser en :

$1^{\circ}$ Relations avec l'Atlantique;

$2^{\circ}$ Navigation fluviale;

$3^{\circ}$ Chemin de fer;

$4^{\circ}$ Postes et télégraphes;

$5^{\circ}$ Routes terrestres;

$6^{\circ}$ Moyens de transports.

\section{Relations avec l'Atlantique}

Sept voies différentes :

A) Dakar à Ouagadougou viâ Saint-Louis, Ambidédi, Kayes, Bamako.

B) Dakar à Ouagadougou, viâ Thiès-Tambacounda, Kayes, Ba mako.

G) Conakry à Ouagadougou, viâ Kouroussa, Bamako.

La première de ces voies présente cet inconvénient grave que le Sénégal n'est accessible aux bateaux à vapeur de faible tonnage que do juillet à novembre et aux vapeurs de haute mer d'août à septembre seulement. 
Jusqu'à l'achèvement du railway Thiès-TambacoundaKayes dont les travaux seront véritablement terminés en 1923, la jonction entre le point terminus et Kayes est assurée par un service de voitures automobiles.

De Bamako à Ouagadougou, plusieurs itinéraires peuvent être suivis : la route de Boug:ouni-Sikasso-Bobo-Dégougou (740 kms) est généralement empruntée. Le Niger peut être également utilisé jusqu'à Ségou ou Mopti; de Ségou, la route passe par San-Dédougou-Koudougou-Ouagadougou ou San-DédougouYako-Ouagadougou; enfin de Mopti la route passant par Bandiagara-Ouahigouya-Yako conduit à Ouagadoug:ou. L'intérêt de chacune de ces voies dépend du point de destination proposé.

D) Accra (Gold Coast) à Ouagadougou viâ Koumassie.

Cette voie emprunte le chemin de fer d'Accra à Koumassie puis la route de Koumassie à Ouagadougou (550 kms environ).

E) Lomé (Togo) à Ouagadougou par Atakpamé.

Cet $_{\text {te }}$ voie emprunte le chemin de fer d'Accra à Atakpamé, puis la route d'Atakpamé à Ouagadougou ('780 kms).

F) Cotonou (Dahomey) à Ouagadougou par Porto-Novo.

Cette voie emprunte le chemin de ier du Dahomey de Cotonou à Agouagou, puis la route d'Agouag:ou à Djougou et SansanéMango (800 kms).

G) Grand-Bassam (Côte-d'Ivoire) à Ouagadougou par Bouaké. Cette route paraît être la route de l'avenir .Le passager débarque à Bassam, prendi la chaloupe jusqu'à Abidjan (prix du passage : 20 francs pour les européens, 10 francs pour les indigènes; prix du frêt : 12 francs au mètre cube ou aux $1.000 \mathrm{~kg} s$ ), d'où dans la même journée, le train le conduit à Bouaké(316 kms) Le service automobile le transporte ensuite en quatre jours de Bouaké à Ouagadougou (805 kms).

Ces délais sont d'ailleurs susceptibles de réduction à brève échéance : dans cet ordre d'idées, il est bon de noter qu'une nouvelle section de $60 \mathrm{kms}$ sera ouverte à l'exploitation dans le courant de 1922, et que l'avancement des travaux se poursuit activement.

\section{Navigation fluviale}

Les deux seules artères fluviales de la Hautc-Volta sont le Niger et la Volta Noire.

Le Niger n'est navigable dans la partie supérieure que pen- 
dant la saison des hautes eaux, des rapides en obstruant le cours pendant la période de la décrue. L'utilisation de ce bief n'en sera pas moins intéressante dès que la rail du Dahomey aura été prolongé jusqu'au fleuve, principalement pour toutes opérations commerciales ayant pour objectil's les Cercles de Dori et de Say.

Les rives de la Volta Noire, infectées par la Tetsé, nécessiteront un aménagement spécial, le déboisement des berges, qui aura pour conséq uence la disparition de la mouche. Un balisage sommaire sera également indispensable. Cette double opération réalisée, cet affluent deviendra utilisable comme voie de communication intérieure et ultérieurement sans doute comme moyen dlévacuation des produits locaux vers l'extérieur.

\section{Chemin de fer}

La Haute-Volta ne possède aucun chemin de fer. Sa situation économique à cet égard se trouve aujourd'hui nettement défavorable. Un avenir très prochain lui ménage par contre une large compensation en lui créant une place privilégiée parmi les colonies du groupe. Par sa position géographique, la Volta est appelée à ètre le point die jonction de toutes les lignes aussi bien étrangères que françaises. Le rail du Dahomey la touchera dans le nord par Say et dans l'est par le tronçon de Djougou prolongé; le Thiès-Kayes et le Kayes-Niger, soudés à Bougouni puis avancés dans la direction de Sikasso-Bobo, pourront devenirdes portes de sortie surl'ouest. Le cheminde fer du Togo sera un débouché sur le sud. Il reste enfin les deux chemins de fer de la Còte-d'Ivoire et de la Gold Coast dont les travaux poussés très activement permettent de prévoir la pénétration imminente de notre hinterland.

La boucle du Niger sera donc desservie un jour par sept chemins de fer qui lui offriront des débouchés faciles dans les directions les plus différentes, lui créant ainsi une situation économique particulièrement avantagée.

\section{Postes et Tëlégraphes}

Lo fil télégraphique relie le chef-lieu de la colonie aux colonies limitrophes et aux cercles. Le réseau doit être complété incessamment et la liaison établie avec les Subdivisions qui, en Haute-Volta, ont l'importance de grands Cercles. Le téléphone sera installé cette année-même dans la ville de Ouagadougou. 
Le service postal et télégraphique est assuré présentement par onze bureaux dont les principaux sont ouverts à toutes les opérations. Des courriers réguliers mettent la colonie en relation directe avec :

$1^{\circ}$ La Côte-d'Ivoire par courrier partant de Bobo-Diousano les 11 et les 28 de chaque mois;

$2^{\circ}$ Le Togo par courrier partant de Ouagadougou le 2 de chaque mois;

$3^{\circ}$ Le Soudan français et Dakar par courrier partant de Ouagadougou chaque quinzaine, le mercredi;

Enfin Ouagadougou correspond avec tous les points de la colonie par courriers réguliers bi-mensuels.

Un arrêté local du 22 mai 1920 détermine les tarifs applicables aux colis-postaux échangés à l'intérieur de la colonie.

\section{Roules lerrestres}

A l'intérieur de la colonie les routes constituent présentement les seules voies de communication.

Un arrêté du 17 mars 1920 les classe en quatre catégories et fixe la largeur des emprises domaniales qu'elles comportent :

$1^{\text {re }}$ catégorie. - Route intercoloniale reliant Ouagadougou aux colonies voisines, largeur d'emprise 25 mètres;

$2^{\circ}$ catégorie. - Routes coloniales reliant directement les chefslieux des cercles; largeur d'emprise 20 mètres;

$3^{\circ}$ catégorie. - Routes de cercles non comprises dans les deux premières catégories, reliant les chefs-lieux de subdivisions entre eux et au chef-lieu de cercle; largeur diemprise : 25 mètres;

$4^{\mathrm{e}}$ catégorie. - (Chemins vicinaux) Tous chemins non compris dans les trois premières catégories; largeur d'emprise : 10 mètres.

Le réseau routier intérieur de la colonie, accessible aux automobiles, mesure $3.000 \mathrm{kms}$ environ. L'administration s'est atta chée à améliorer les voies existantes, déjà réputées par leur praticabilité, leur largeur, l'excellence de leur tracé, le bon entretien de leur chaussée.l'existence de ponts en terre durcie pour la traversée de tous les marigots.

Des campements, distants en moyenne de $25 \mathrm{kms}$, toujours situés à proximité d'un village, donnent aux voyaģeurs un abri suffisant et la facilité d'un ravitaillement en eau et en denrées de première nécessité (lait, œufs, poulets, mil, etc.), 


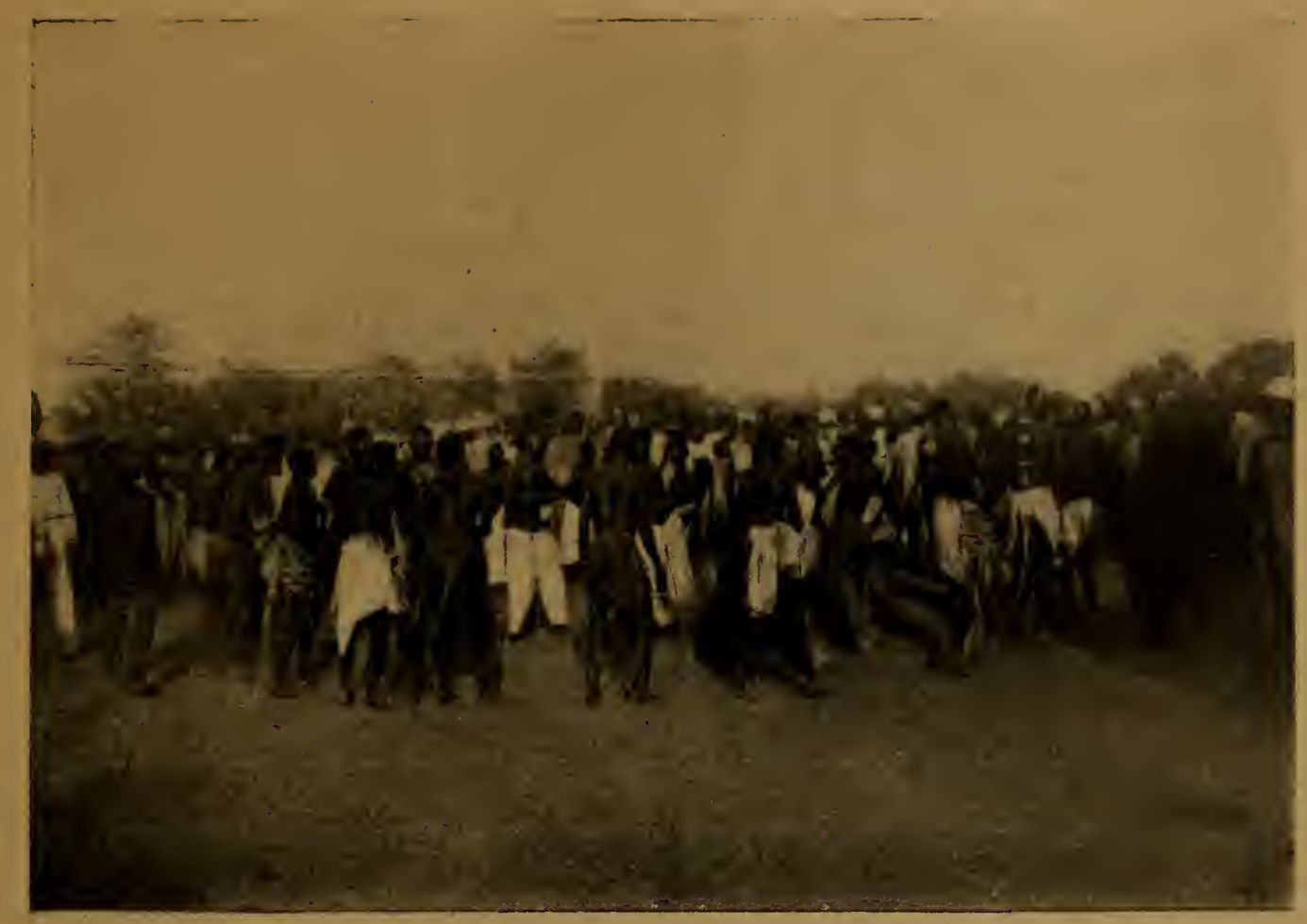

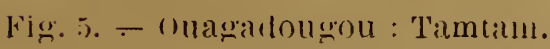

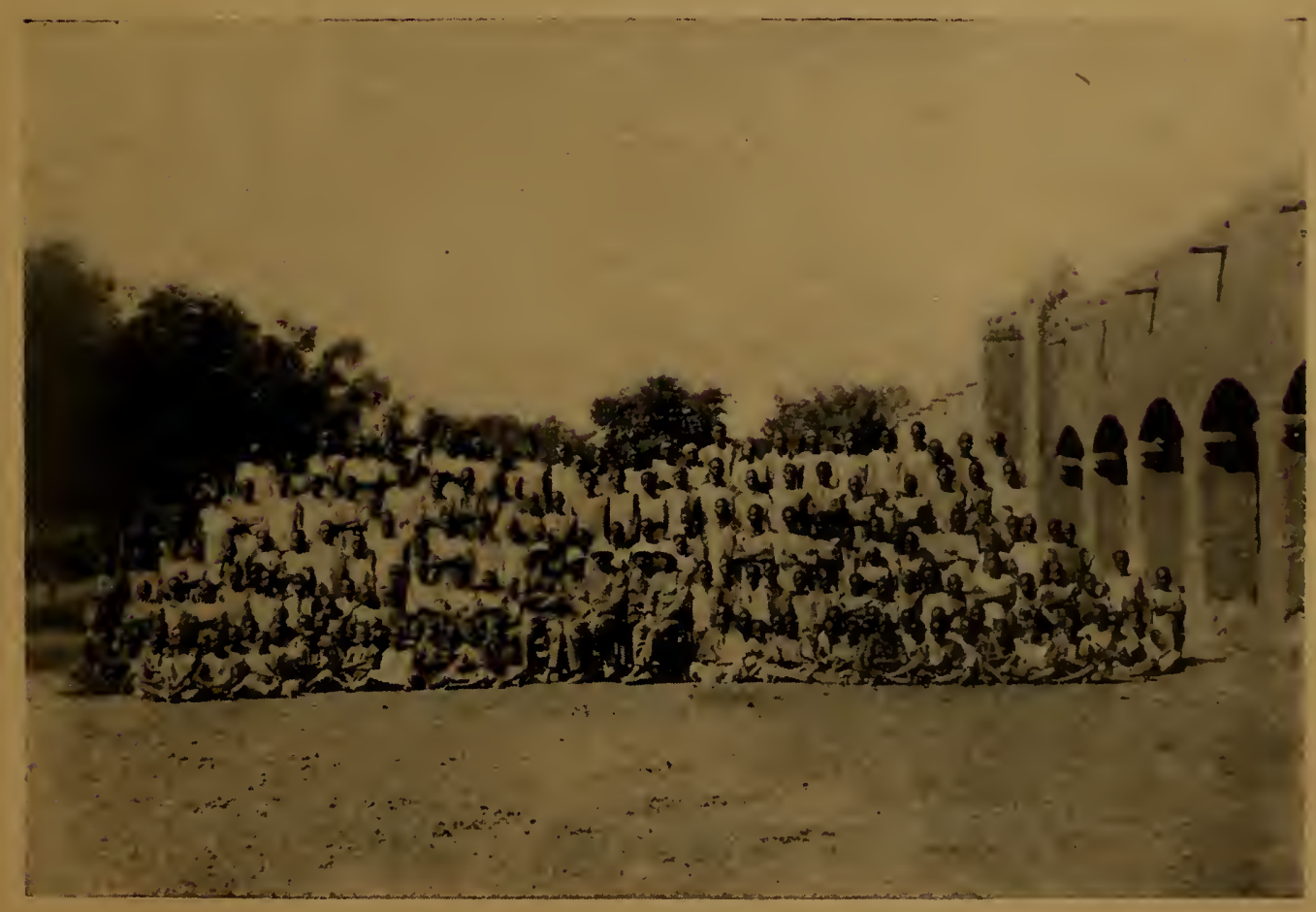

Fig. 6. - Ouagadougou : Ecole régionale. 



\section{Moyens de Transports}

Les moyens de transports en usage dans la colonie sont de quatre sortes différents :

$1^{\circ}$ Le portage à tête d'homme règlementé par l'arrêté local du 23 novembre 1920 qui détermine les allocations, les étapes et le proids des charges;

$2^{\circ}$ Le portage par animaux fait l'objet des arrêtés locaux des 6 juillet et 25 novembre 1920.

Le premier de ces actes, inspiré par des considérations humanitaires qui tendent à la suppression du portage humain, a créé dans les cercles des corporations d'âniers subventionnées;

$3^{\circ}$ Une autre amélioration des moyens de transports, aujourd'hui en voie d'exécution, consiste dians la création d'un matériel de roulage représenté par la charrette à bœufs du type usité en Algérie et à Madagascar. A cet effet la colonie a engagé un maître-charron européen qui, sous le contrôle du service des travaux publics dirige à Ouagadougou une école de charronnage. Un matériel et un outillage d'une valeur de 30.000 francs sont déjà en place.

La charrette tirée par deux bœufs sera utilisable pour une charge de $500 \mathrm{kgs}$ environ.

$4^{\circ}$ Le service des automobiles, créé en 1920, est destiné essentiellement à assurer la liaison entre le terminus du rail de la Côte-d'Ivoire et la capitale de la colonie. La relève du' personnel et le transport du matériel s'effectuent par cette voie. L'existence de ce service nécessitera la création, dès 1922, d'un deuxième garage à Bobo-Dioulasso qui sera placé comme celui de Ouagadougou sous la direction d'un mécanicien européen. 


\section{CHAPITRE V}

\section{Productions du sol}

\section{Cullures vivrières}

Le petit mil est la base de l'alimentation indigène : la production annuelle largement suffisante aux besoins de la population peut être estimée à 1.100 .000 tonnes. Le prix du kilogramme varie de 0,07 dans la brousse à l'époque de la moisson à 0,75 au moment de la soudure ou à 1 franc quand la récolte a été déficitaire.

Le gros mil ou sorgho, utilisé pour la nourriture des chevaux et la fabrication de la bière indigène, a un rendement annuel moyen de 200.000 tonnes.

Le riz (17.000 tonnes) cultivé de façon assez intensive notamment dans le cercle de Tenkodogo se vend de 0 fr. 50 à 1 fr. 25 le kilo. Les surfaces susceptibles d'ètre transformées en rizières sont considérables. La culture du maïs prend une extension chaque année plus grande : le prix de cette denrée oscille entre $0 \mathrm{fr} .30$ et $0 \mathrm{fr} .50$ le kilo. Le fonio est connu surtout dans la région du nord.

Le manioc reste assez peu répandu. L'ensemble de ces quatre cultures fournit approximativement 20.000 tonnes par an.

Les prois ne représentent qu'une denrée: de complément.

Les haricots dits " kissi " semés avec les premières pluies d'hivernage sont particulièrement avantageux tant par la série des nombreuses récoltes qu'ils donnent que par leur rendement exceptionnel.

L'arachide, dont la culture est exclusivement aux mains des femmes, n'occupe que des surfaces très restreintes. Les plaines de la Haute-Volta se prêteraient très bien à la production de l'arachide qui prendra incessamment un développement appréciable en raison de l'installation d'une huilerie à Ouagadougou.

Le ricin, dont la culture préconisée pendant la guerre avait 


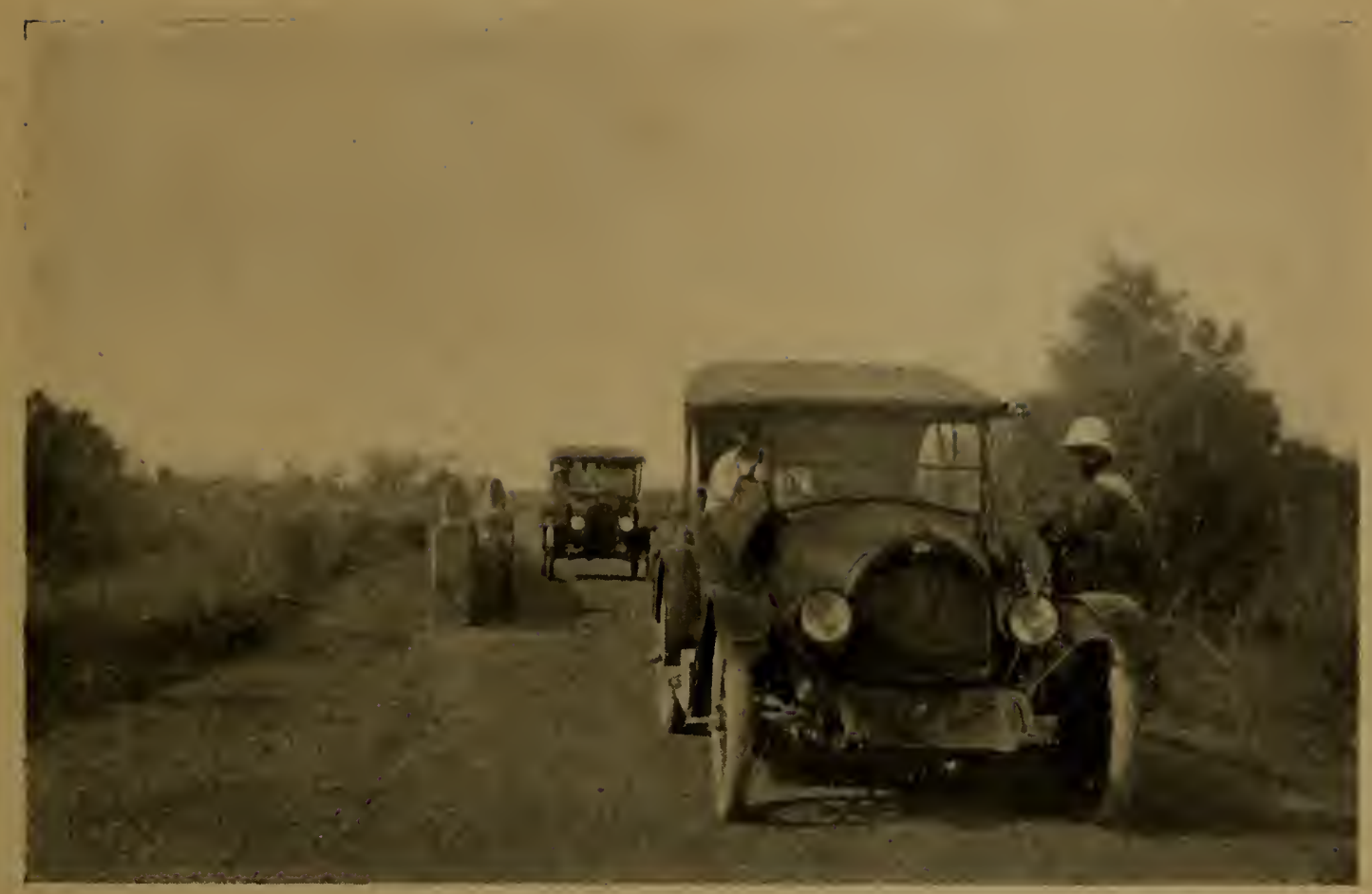

Fig. 7. - Routes de la Haute-Tolta. Autos flanchissant un pout.

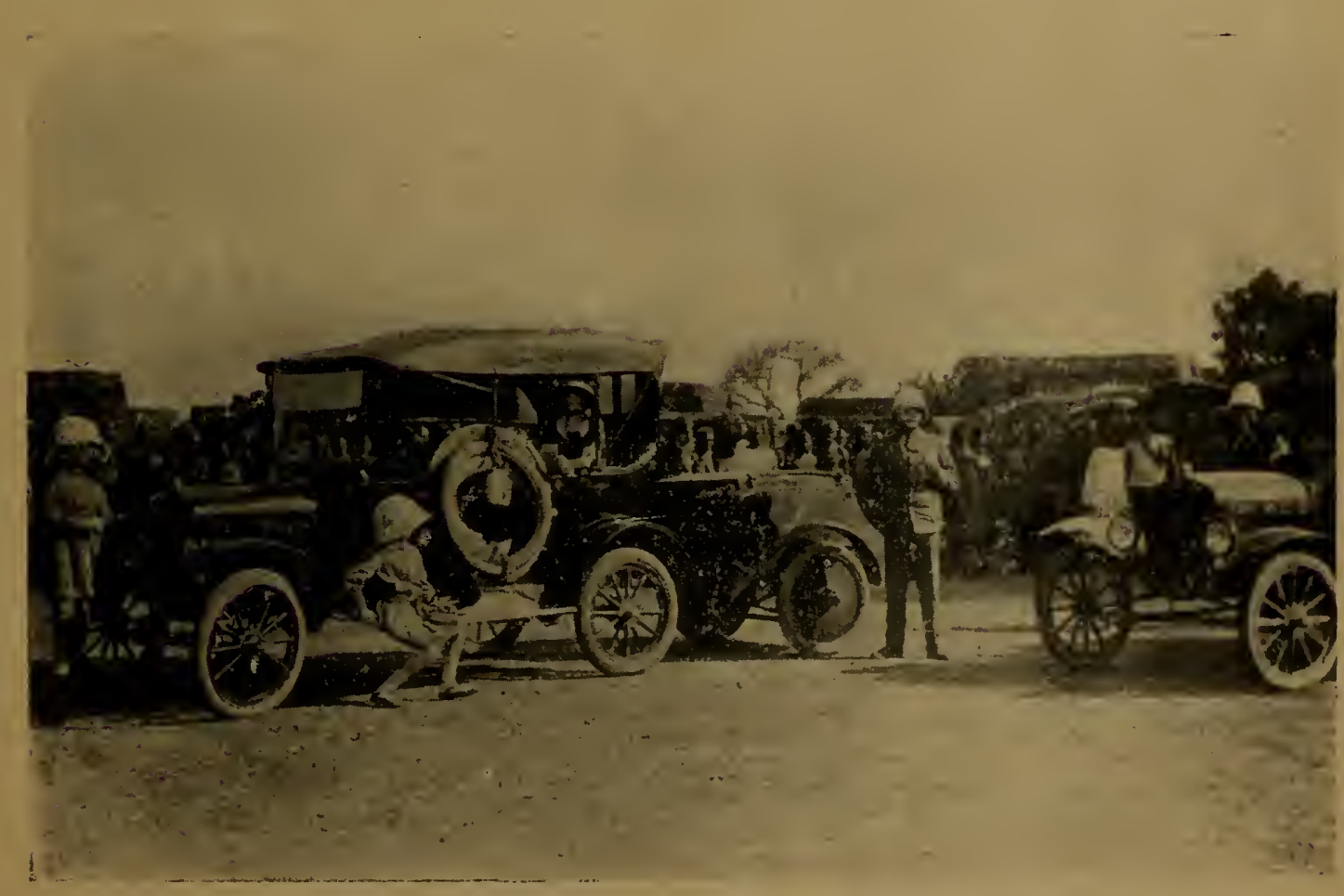

Fig. s. - Arrêt d'un convoi d’automobiles dans un rillaçe.

HAUTE-VOLTa, - PLANCBE IIII 

donné d'excellents résultats est aujourd'hui abandonné. Il serait facile d'y intéresser les indigènes à la condition de leur garantir des débouchés.

Sous l'impulsion de l'administration qui répartit des semences entre les collectivités, la pomme de terre autrefois localisée aux environs de Sikasso s'est étendue d'abordl à Bobo-Dioulasso, puis aux autres cercles de la colonie. La circonscription de Bobo, sur une récolte totale de 12 tonnes, après avoir satisfait aux exigences de la consommation locale a pu, en 1921, exporter 7 tonnes en Côte-d'Ivoire.

Des essais de blé dur ont été tentés pour la première fois en 1921 sur les terrains administratifs des cercles; le rendement a été approximativement de 13 pour un. Ces cultures intéressantes au premier chef vont être intensifiées et un moulin sera installé à Bobo-Dioulasso.

Chaque famille indigène a son champ de tabac et ce produit donne lieu à un trafic sérieux. Mais les procédés rudimentaires de culture rendent à peu près inutilisables les feuilles récoltées. Des plantations créées par des Européens et notamment à Dédougou au moyen de graines " mélia " ont donné des plants de 2 mètres et plus dont les plus belles feuilles sont de 15 à 20 fois plus grandes que celles du tabac indigène.

\section{Produits industriels}

La question du coton est plus que jamais à l'ordre du jour en A. O. F. et des travaux considérables destinés à alléger la charge dont, à ce titre, nous sommes tributaires envers l'étranger, se poursuivent actuellement dans la vallée du Niger. Sa situation géographique favorable et la densité de sa population commandent à la Haute-Volta de ne point demeurer indifférento à l'effort qui se précise pour l'alimentation de nos filatures nationales. Les études et les expériences des spécialistes en la matière ont conduit à cette double conclusion : abandon du plant indigène peu susceptible d'amélioration et adaptation au pays d'espèces américaines hâtives auxquelles seront appliquées les méthodes culturales déjà en usage, perfectionnées au moyen d'un système d'irrigation. En dehors des deux cours d'eau permanents de la colonie : Niger et Volta Noire, la question d'eau qui de prime-abord peut paraître aiguë dans laboucle sera heu- 
reusement résolue par l'établissement de barrages. La production moyenne des dernières années peut être évaluée à un millier de tonnes de coton brut et ce rendement est suffisant pour justifier l'installation à Ouagadougou d'usines: d'égrenage dont le montage sera terminée en 1922 .

L'exploitation du caoutchouc ne peut être pratiquée que dans les cercles du sud. Les peuplements ont été reconstitués et la reprise des cours semble devoir encourager une opération dont l'intérêt se manifestera surtout avec l'avancement du rail de la Côte-d'Ivoire.

Le Dâ (ou chanvre de Guinée) dont le rendement est environ de 1.600 kilos de fibres à l'hectare pourrait justifier de vastes exploitations très rémunératrices tant à cause de la simplicité des méthodes de culture que de la possibilité de le traiter par des moyens mécaniques.

L'absence de moyens de transpörts ne permet pas l'utilisation du kapok que la colonie produit en quantité considérable.

\section{Elevage}

L'élevage constitue sans doute la richesse la plus importante de la Haute-Volta; il est pratiqué principalement dans les cercles du nord, Say, Fada et surtout Ouahigouya et Dédougou où se trouve l'habitat des Peuhls. Mais le climat de la colonie per. met le développement de l'élevage sur toute l'étendue de son territoire.

La race chevaline ne comporte qu'un seul type dont les caractéristiques le rapprochent de celles de la race mongolique. La Haute-Volta possède plus de 30.000 chevaux d"une valeur moyenne de 300 francs, mais les beaux sujets dépassent ce prix et atteignent jusqu'à 1.000 francs. De $1 \mathrm{~m}$. 45 à $1 \mathrm{~m}$. 55 à Dori la taille des chevaux tombe à $1 \mathrm{~m}$. 35 et même à $1 \mathrm{~m}$. 28 à BoboDioulasso.

Les asinés d'origine nord-africaine sont de petite taille mais robustes. Ils portent aisément 60 kilos. Il y a environ 65.000 ânes dans la colonie et le prix de l'unité oscille entre 80 et 130 francs.

Les bovidés sont de deux espèces : au nord le zébu ou bœuf à bosse dont la taille atteint $1 \mathrm{~m} .35$ à $1 \mathrm{~m} .45$ au garrot et dont la bosse peut atteindre de 10 à 15 centimètres de haut. 
Au sud de la colonie, vit le bœuf sans bosse dit du Fouta-Djallon : il a environ $1 \mathrm{~m} .10$ de haut. Malgré les épizooties qui sévissent périodiquement, le cheptel peut être estimé à 400.000 bovidés qui donnent lieu à un mouvement commercial important avec la Gold Coast. Acheté sur place, moyennant une somme variant de 100 à 160 francs, le bœuf se vend à Koumassie jusqu'à 16 et 20 livres.

Le nombre des ovidés et des capridés dépasse un million cent mille : le prix moyen va ide 8 à 25 francs suivant la taille et la qualité. Les moutons dits de "case " atteignent jusqu'à 50 et $75 \mathrm{fr}$. Cet aperçu des prix correspond aux cours actuels et à la hausse résultant de l'état de guerre. La question de l'élevage est une des celles qui intéresse au plus haut point i avenir de la HauteVolta; elle est complexe et nécessitera aussi bien l'intervention des administrateurs que celle des hommes die l'art : la création d'un cadre complet de vétérinaires européens, le renforcement du cadre des vaccinateurs indigènes, l'étude des affections spéciales aux régions tropicales, la création de laboratoires, le règlement des transhumances, l'établissement de barragēs destinés à enrichir les pâturages et à abreuver les troupeaux sont autant de détails qui préoccupent l'administration supérieure. La réalisation de ces projets aura pour effet le décuplement à brève échéance du cheptel et la transformation de la colonie en une véritable Argentine africaine.

\section{Produits du sous-sol}

Les ressources minières de la Haute-Volta sont encore assez peu connues et des sondages sérieux seraient nécessaires pour permettre l'évaluation de la valeur du sous-sol.

La principale richesse est représentée par les placers du Lobi qui donnent un rendement moyen de 50 kilos d'or par an. Mais ii y a lieu de considérer que l'exploitation est entièrement aux mains des indigènes et que les procédés d'extraction employés sont encore tout-à-fait primitifs. L'exemple de la Guinée doit nous inciter à être provisoirement très réservés sur cette question.

Le minerai de fer est abondant et notamment dans le nord de li colonie. Il est exploité par les forgerons indigènes. L'argile et le kaolin sont également abondants. 
Le Cercle de Say possède une petite exploitation de jaspe dont se servent les forgerons pour la confection de bijoux à l'usage des femmes indigènes.

De l'énumération de ces produits il ressort que la Haute-Volta est essentiellement une région agricole et un pays d'élevage. Son sol lui procure très largement tout ce qui lui est utile pour assurer le bien-ètre de ses habitants. La colonie n'a besoin d'aucun appel à l'extérieur : elle est déjà un pays d'exportation. Ce mouvement doit prendre une amplitude toujours plus grande avec l'augmentation de la population, le développement des débouchés et toutes les mesures qu'il nous appartient d'envisager pour favoriser l'essor éconnmique de cette riche possession.

\section{CHAPITRE VI}

\section{La Colonisation agricole, industrielle et commerciale}

\section{Colonisation Agricole}

La colonisation agricole européenne n'est représentée jusqu'à ce jour dans la Haute-Volta que par deux concessions rurales de 900 hectares chacune accordées aux environs de Ouagadougou à la mission des Pères Blancs d'Afrique. Dans l'une d'elle, à Fabré, a été établi un important barrage d'une capacité de plusieurs millions de mètres cubes et destiné à l'irrigation des cultures maraîchères et des vergers; une plantation dø quelques milliers de Teks obtenus au moyen de graines importées du nord de la Gold Cioast et dont certains sujets atteignent déjà 5 à 6 mètres de hauteur constitue une expérience intéressante dans un pays où le bois d'œuvre trouvera un emploi de plus en plus grand.

\section{L'Industrie}

Pour la première fois, en 1922, l'industrie européenne sera représentée à Ouagadougou par une usine électrique, une machine à glace, une huilerie et une scierie mécanique. Ces installations payées sur les fonds du budget local ont pour fin l'éclai- 
rage du chef-lieu, la fourniture de glace à l'hôpital ainsi qu'à la population européenne et le sciage du bois pour le service des travaux publics.

Une mention doit être accordée à l'atelier dirigé par les religieux de la mission catholique qui fabrique des tapis de laine très appréciés, du même type que celui usité en Algérie.

Les industries sont identiques à celles des colonies voisines : tissage de bandes de coton, travail du fer, teintureries, cordonneries, vanneries, poteries, etc. La plus importante, sans contredit, est celle du tissage de bandes de coton d'une largeur de 0,10 à 0,20 , pratiquée au moyen du métier primitif qui se rencontre dans toute l'Afrique Occidentale. Ces bandes de coton donnent lieu à un mouvement commercial considérable et sont exportées notamment dans la vallée du Niger et en Gold Coast.

Les forgerons extraient par la méthodie catalane leur fer du minerai qui abonde dans tout le pays. L'outillage indigène en entier (haches, hachettes, couteaux, serrures, gonds, clous, instruments agricoles, etc.) est fabriqué avec les ressources et les moyens propres du pays.

Par des procédés assez rudimentaires, les cordonniers tannent les peaux provenant de l'abattage des animaux et confectionnent divers objets, chaussures, sacoches, selles,porte-monnaie, coussins, etc.

La vannerie est aux mains des Peuhls qui utilisent la fibre des agaves et de certaines plantes de marais pour la fabrication de sacs, d'éventails, de chapeaux, de nattes, etc .

Tous ces travaux décèlent parfois du goût et une réelle habileté. La production est insuffisante et aussi trop inégale pour intéresser le commerce européen.

\section{Le Commerce}

Le courant commercial entre la Haute-Volta ét la Gold Coast est particulièrement intense. Les deux principaux articles qui alimentent ce trafic sont, d'une part le bétail de la Boucle qui va approvisionner le marché anolais et d'autre part les noix de kola que les colporteurs importent de la colonie britannique.

Cette situation est gravement préjudiciablè à nos intérêts, en ce sens que nos possessions du Soudan français et de la Côted'Ivoire; pour ne citer que les deux colonies limitrophēs de la Haute-Volta et qui auraient besoin de notre assistance économi- 
que, se voient à peu près délaissées. Cette observation s'applique non seulement au mouvement commercial proprement dit, mais aussi à la main-d'œuvre qui se porte avec une préférence bien marquée vers les plantations de cacao, le chemin de fer ou les mines d'or de la Gold Coast.

Cet état de choses a attiré l'attention de l'administration supérieure qui, après avoir déterminé la cause de l'attraction exercée par la colonie étrangère, étudie les mesures propres à donner une orientation nouvello à notre commerce et à nos travailleurs. Présentement l'autorité anglaise estimait que 100.000 bœufs et 100.000 ovidés représentant un capital de plus $5.500 .000 \mathrm{fr}$. descendent chaque année du Soudan et de la Haute-Volta sur Koumassie. Sur cette somme $70 \%$ rentreraient en territoire français sous forme d'espèces monnayées; $20 \%$ seraient échangés contre divers articles et le reliquat correspondrait aux dépenses d'entretien.

Ces chiffres sont vraisemblablement inférieurs à la réalité.

Si l'on estime à 8.000 .000 francs, chiffrel qui résulte des statistiques officielles, le montant des transactions effectuées entre la Gold Coast et la Haute-Volta, on se rendra compte qu'il représente presque la totalité du commerce de la colonie puisque la Nigéria et l'ensemble de nos grandes possessions n'entrent en ligne, la première que pour 200.000 et les autres pour 300.000 frs. seulement. A ces évaluations, il convient d'ajouter l'importation des articles européens qui s'élèvent approximativement à 1.000 .000 .

Il a déjà été dit que les bovidés et les ovidés alimentent l'exportation 'tandis que les kolas, les tissus et divers articles représentent les principaux articles d'importation. Le commerce intérieur est également actif, il porte principalement sur les denrées vivrières, le tabac, les bandes de coton, le bétail, la poterie, la vannerie, etc.

En prenant pour base le montant de la perception des " droits de marché " les transactions intérieures effectuées chaque année peuvent être estimées à 10.000.000 environ. Elles se décomposeraient ainsi : 3.150 .000 pour les produits d'importation, 350.000 pour les produits exportés sur place et 6.500 .000 pour le trafic purement intérieur. 


\section{Conclusion}

De ce court exposé, il ressort que la nouvelle colonie dont la situation générale est déjà si prospère autorise pour un avenir prochain les plus belles espérances.

La salubrité de son climat qui la rend habitable à l'Européen, la densité toujours croissante de la population qui procurera aux entreprises publiques et privées une abondanto main-d'œuvre, la fertilité de son sol qui offre les ressources les plus variées constituent des garanties certaines de son développement économique.

Ses moyens de communication qui ont été perfectionnés d'une manière sensible depuis la création de la nouvelle colonie deviendront les auxiliaires précieux des futurs tronçons du chemin de fer sur lesquels ils évacueront l'excédent de la production des riches cantons. Le rail n'aboutira sans doute pas à Ouagadiougou avant une dizaine d'années : à cette époque, la colonie entière se trouvera encerclée par l'ensemble des chemins de fer en construction en A. O. F. Il en résultera pour elle une situation tout à fait privilégiée parmi les autres colonies du groupe.

L'extension des cultures dont l'indigène a déjà pu apprécier les bienfaits, l'adaptation au pays des méthodes culturales appropriées aux divers produits, l'établissement de barrages multipliés à l'infini que les habitants ne cessent de demander, l'organisation sur des bases solides d'un service zootechnique pour la conservation et l'amélioration du cheptel, autant de mesures pour ne citer que les principales, qui se trouvent actuellement à l'étude ou en voie d'exécution.

Leurapplication intégrale qui sera l'œuvre d'une période relativement brève qui ne saurait dépasser quelques années est destinée à assurer le plein essor de la Haute-Volta. 


\section{PRINCIPAUX OUVRAGES \\ A CONSULTER SUR LA HAUTE-VOLTA}

E. Jougla.... Bibliographie de l'Afrique Occidenlale. Paris. Sansot, 1912.

Terrier et Mourey. L'Euvre de la Troisième République en Afrique, Occidentale. L'Expansion française et la formation territoriale. Paris, Larose, 1889,

Delafosse.... Haut-Sénégal-Niger (1 ${ }^{\mathrm{re}}$ série). Le Pays, les Peuples, les Langues, l'Histoire, la Civilisation, Paris, Larose, 1912, 3 vol.

J. Meniaud ... Haut-Sénégal-Niger ( $2^{\mathrm{me}}$ série). Géographie économique. Paris, Larose, 1912, 2 vol.

G. Binger.... Du Niger au golfe de Guinée par le pays de Kong et le Mossi, Paris, Hachette et Cie, 1892, 2 vol.

$\mathrm{L}^{\mathrm{t}}$ Marc...... Le Pays Mossi, Paris, Larose, 1908, 1 vol.

A. Le Ghatellier. L'Islam en Afrique Occidentale. Paris, 1889, 1 vol. Hubert....... L'état actuel de nos connaissances sur la Géologie de $l$ 'A. O. F. Paris, Larose, 1920. 

